\title{
Norois
}

Environnement, aménagement, société

$233 \mid 2014$

Mobilité, santé et développement territorial : de nouveaux défis pour la gouvernance des territoires ruraux

\section{Habiter les espaces ruraux : les enjeux des formes de mobilité des jeunes. Regards interdisciplinaires}

Living in rural areas: interdisciplinary perspectives on young people's mobility

Mélanie Gambino et Olivier Desmesure

\section{OpenEdition \\ Journals}

Édition électronique

URL : https://journals.openedition.org/norois/5401

DOI : $10.4000 /$ norois. 5401

ISBN : 978-2-7535-4127-6

ISSN : $1760-8546$

Éditeur

Presses universitaires de Rennes

Édition imprimée

Date de publication : 20 décembre 2014

Pagination : 25-35

ISBN : 978-2-7535-4083-5

ISSN : 0029-182X

Référence électronique

Mélanie Gambino et Olivier Desmesure, « Habiter les espaces ruraux : les enjeux des formes de mobilité des jeunes. Regards interdisciplinaires », Norois [En ligne], 233| 2014, mis en ligne le 20 décembre 2016, consulté le 14 janvier 2022. URL : http://journals.openedition.org/norois/5401 ; DOI : https://doi.org/10.4000/norois.5401 


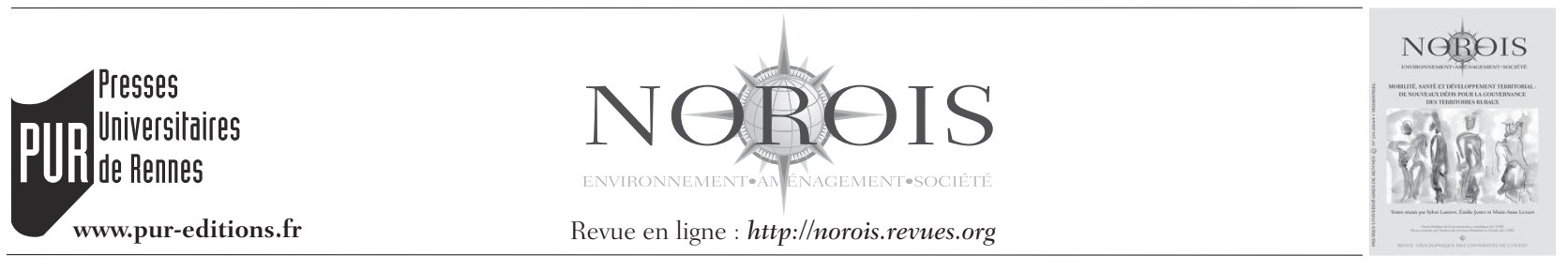

\title{
Habiter les espaces ruraux : les enjeux des formes de mobilité des jeunes - Regards interdisciplinaires
}

\author{
Living in Rural Areas: Interdisciplinary Perspectives on Young People's Mobility
}

Mélanie GAmbino $^{\text {a*}}$, Olivier Desmesure ${ }^{\mathrm{b}}$

\footnotetext{
* Auteur correspondant

a UMR Dynamiques Rurales (MA 104) - Université de Toulouse II Jean Jaurès, 5, Allées Antonio-Machado - 31058

Toulouse Cedex 9, France (gambino@univ-tlse2.fr)

${ }^{\mathrm{b}}$ Conseiller jeunesse DDCSPP Dordogne; Centre de recherche en médecine et psychanalyse (EAD n 3522$)-$

Université de Paris VII Diderot, 5 Rue Thomas-Mann - 75013 PARIs, France. (olivier.demesure@dordogne.gouv.fr)
}

Résumé : En s'appuyant sur des éléments de réflexion issus de plusieurs recherches dans des disciplines peu habituées à travailler ensemble, la géographie et la psychopathologie, nous livrons une analyse sur une partie mal connue de la jeunesse contemporaine, les jeunes de 15 à 25 ans qui vivent dans les espaces ruraux de faible densité de population. À partir de deux études de cas internationales (en France et en République d'Irlande), nous considérons, en prenant pour objet les modes d'habiter, que les mobilités des jeunes sont désormais plurielles. Elles se déclinent sur le mode de la circulation, de l'ancrage ou de l'errance. Elles trouvent leur origine dans leur rapport au territoire et dans les formes que les jeunes définissent à leur trajectoire.

\begin{abstract}
Based on different studies in two foreign disciplines (geography and psychopathology), this article focuses on a poorly documented part of today's youth population: 15 to 25 year old young people living in sparsely populated areas. In France and in the Republic of Ireland, where our cases studies are set, we explain how young people's mobility is nowadays plural. We show that the mobility of young people, often unexpected, can be qualified as circular, sedentary or close to roving life. It originates from their territoriality and how they define their trajectory.
\end{abstract}

Mots clés : habiter - jeunesse - espace ruraux de faible densité - pratiques spatiales - représentations sociales

Keywords: to inhabit - youth-sparsely populated areas - spatial practices - social representations

\section{INTRODUCTION}

La jeunesse rurale en tant que stéréotype renvoyant à la société paysanne n'existe plus. Ce groupe a subi de nombreuses transformations : la jeunesse rurale n'est pas la jeunesse agricole et présente des caractéristiques populaires et ouvrières
(Galland, Lambert, 1993). De plus, les modes de vie, les valeurs et les loisirs tendent à s’homogénéiser entre jeunes urbains et jeunes ruraux. Cela ne signifie pas pour autant qu'il n'y a plus de jeunes dans les espaces ruraux. Mais, comme le souligne N. Renahy (2005), bien souvent la jeunesse rurale 
apparaît reléguée par rapport à celle des espaces urbains, davantage médiatisée. Les jeunes ruraux ont, en outre, le sentiment d'être moins considérés que ceux vivant dans des espaces urbains (Bronner, 2007). La récente crise économique a renforcé ce sentiment, d'autant plus que les espaces ruraux de faible densité offrent peu d'opportunité d'emploi et des conditions de travail qui n'attirent pas toujours les jeunes, ce qu'ils intériorisent fortement. L'objectif de cet article est donc de porter une analyse pluridisciplinaire sur cette partie mal connue de la jeunesse d'aujourd'hui, en explicitant les modes d'habiter adoptés, notamment à travers la mise en relation entre leurs pratiques spatiales et leurs représentations sociales de la ruralité. L'analyse des modes d'habiter, du point de vue du géographe ou du point de vue psychopathologique permet également de mettre au jour une mobilité des jeunes peu repérée mais qui n'a de cesse d'exprimer «les contretemps de la jeunesse »(Desmesure, 2011): ils sont appréhendables à partir d'un mouvement spontané qui n'est pas ou peu pris en compte dans ce qu'on appelle communément la mobilité. Or, il s'agit là d'un versant singulier de la mobilité qui dérange lorsqu'on le revendique comme moteur de la construction d'un parcours ou d'une trajectoire. La mobilité, lorsqu'elle s'apparente à un tâtonnement, est décrite comme une perte de temps par les pouvoirs publics et les adultes, que l'on soit en situation d'entrée dans le monde du travail ou sur le «marché scolaire » alors qu'elle représente, selon nous, une opportunité pour la jeunesse d'être là où on ne l'attend pas, ce qui est en soi une forme de mobilité inédite et peu analysée. Nous illustrons ici que pour les jeunes, l'apprentissage et l'exercice de la mobilité ne sont pas linéaires et ne vont pas de soi. Ils se font par à-coup, par des contretemps qui peuvent nous saisir à bien des égards de par leur effet de surprise. L'objectif ici est donc bien de donner à voir une jeunesse qui n'est jamais là où on l'attend et de cerner les enjeux pour les espaces ruraux de cette mobilité imprévisible.

\section{« L'HABITER », POINT DE DÉPART D'UNE ANALYSE PLURIDISCIPLINAIRE DES MOBILITÉS DES JEUNES}

Pour tenter de définir un cadre commun à la pratique interdisciplinaire, nous sommes partis d'une définition ouverte de "l'habiter » initiée dans les travaux de M. Gambino (2008) et dont le regard pluridisciplinaire s'est exprimé grâce à l'influence de M. De Certeau (1990). Nous considérons que l'habiter est un acte complexe et ne se réduit pas à une simple occupation des lieux. Il implique à la fois des comportements, habitudes, sentiments, représentations qui se matérialisent dans les pratiques des jeunes. Cette définition passerelle intègre une complexité mais elle a le mérite, pour ce qui nous concerne, de montrer les limites propres à nos modèles théoriques. L'approche géographique est bénéfique quand elle concentre son analyse sur les « représentations » et les « habitudes » des jeunes et celle de la psychopathologie enrichie l'analyse lorsqu'elle prend plus de temps pour établir un rapport entre l'habiter, les pratiques sociales et les «affects » des jeunes.

Mais, au-delà de cette complémentarité, nous avons repéré un fil conducteur entre ce que dit M. De Certeau au sujet de l'habiter comme prise en compte de "l'insignifiant et de l'anecdotique » et ce que nous apprend l'anthropologie comparative au sujet du mythe comme expérience, « une expérience mythique » (Détienne, 1981), des expérimentations revendiquées maladroitement par les jeunes lorsqu'ils réhabilitent des «mondes retranchés ». Ce que la géographe décrit comme étant des « recoins » (Gambino, 2011) pour les jeunes et ce que le clinicien appelle « les arrières boutiques » (Desmesure, 2011).

Le propos de M. De Certeau (1990) invite à s'intéresser à la dynamique qui fonde l'habiter. Pour nous, elle s'appuie sur « deux pulsions antagonistes et complémentaires » (Guetat-Bernard, 2007), la première étant liée à l'abri, la protection, la stabilité, l'enracinement et la seconde étant motivée par la découverte, l'aventure, la mobilité, le dépassement et dans certains cas, la métamorphose. C'est pourquoi notre intérêt se porte si fortement sur l'habiter. Ce n'est pas une simple occupation des lieux mais un acte (Lazzarotti, 2006) qui implique à la fois des comportements, habitudes, représentations, affects qui se matérialisent dans les pratiques. Cet acte est constitué de lieux et de liens entretenus par le travail, par la filiation, la mémoire, la projection dans le futur. C'est un acte géographique qui inscrit les hommes dans l'espace à toutes les échelles (Besse, 2013) et plus globalement, c'est une expérience de 
mise à l'épreuve, un « passage », qui laisse des traces dans le territoire. Comprendre cette expérience de l'habiter passe donc par un repérage des pratiques sociales et spatiales où « se trament en effet les conditions déterminantes de la vie sociale »(De Certeau, 1990) pour relever les logiques d'usages et d'appropriations de l'espace, qui ne sont pas dans le cas des jeunes, comme les adultes ou les acteurs publics voudraient qu'elles soient.
On ne peut pas comprendre l'analyse pluridisciplinaire présentée ici si on ne la replace pas dans un contexte de passerelle entre un «espace vécu » qui, par définition, est bien celui de la géographie et celui psychopathologique qui a trait à un « arrière monde » du jeune, dont on va voir paradoxalement qu'il pèse, de par sa réalité, sur sa trajectoire.

\section{Enquêtes et méthodes}

L'analyse présentée ici utilise un corpus d'enquêtes issu de deux recherches achevées s'intéressant aux conditions dans lesquelles la ruralité est aujourd'hui habitée par les jeunes.

Les travaux de M. Gambino s'appuient sur un doctorat de géographie (Gambino, 2008) portant sur les pratiques et les représentations des espaces de faible densité. La méthode d'enquête s'appuie sur l'observation participante, la réalisation et l'analyse de 100 entretiens semi-directifs individuels avec des jeunes de 15 à 25 ans vivant en France (Périgord Vert) et en Irlande (partie rurale du comté de Galway). Les jeunes ont été choisis pour la diversité de leur trajectoire personnelle et de leur position sociale (membre d'une association, lycéens, jeunes parents, en recherche d'emploi...). Tous vivent depuis plus de 5 ans dans les deux zones d'étude. En dépit des différences des contextes nationaux, de fortes similitudes ont été constatées entre les deux pays.

Les travaux d'O. Desmesure (2011) sont issus d'un doctorat de psychopathologie. Les jeunes enquêtés sont situés dans le département de la Dordogne et ils ont fait l'objet d'un premier repérage par le biais de leur prise d'initiative dans la vie publique locale. L'intérêt de ce point d'observation réside dans la connaissance longitudinale de trajectoires de jeunes, avant, pendant et après la rencontre. Ce travail est le saisissement d'un point de vue analytique élaboré entre le jeune et une clinique de l'éducation. Le terme même de « clinique de l'éducation » fait référence à un corps de recherches issu de la tradition psychanalytique et désigne le « lieu de théorisation où des connaissances se construisent à même le vivant et dans l'implication » (Cifali, 1996). Cette clinique qui ne néglige ni les « savoirs savants » ni la « singularité du vivant » s’incarne aujourd'hui grâce aux nombreux travaux dont ceux du Centre de recherche sur la clinique de l'éducation à l'université de Paris 8. Il s'agit donc de partir d'une initiative portée par un jeune tout en se donnant, avec celui-ci, la possibilité de revenir à «l'expérience source » (Boccara, 2002) et ses fondements affectifs. En quelque sorte, il existe bien un fond pulsionnel à l'action mais c'est par le biais de l'affect, en tant que «connaissance » (Bion, 1967), que nous reconstituons, avec le jeune, ce que nous considérons comme une trajectoire et donc un déplacement (voire une mobilité) pas comme les autres : autant, en effet, nous voyons bien ce que le jeune met en jeu lorsqu'il exécute son initiative, autant, notre méthode de travail est édifiante pour enregistrer ce que nous appelons «l'envers du décor ». Cette méthode s'appuie principalement sur des séries d'entretiens cliniques, des observations et une connaissance chronologique du jeune.

Différentes définitions des « espaces ruraux de faible densité » existent, tant le critère démographique est relatif. Ce sont des espaces qui ont été désignés par des termes à connotation négative : le rural «profond », la « diagonale du vide »... Ici, nous faisons référence à des espaces de moins de 40 habitants $/ \mathrm{km}^{2}$.

Le point de vue méthodologique compréhensif adopté lors de la réalisation de ces recherches invitent à souligner qu'il ne s'agit pas de prétendre à une comparaison entre elles mais bien de faire exister des parcours, des discours et des figures d'un « vécu » des espaces ruraux de faible densité. 


\section{LES MODES D'HABITER DANS LES ESPACES RURAUX AUJOURD'HUI : ENTRE CAPTIVITÉ ET ANCRAGE}

L'approche géographique des modes d'habiter des jeunes a mis en évidence trois groupes qui ont chacun une représentation des espaces ruraux et des pratiques.

\section{Ruralité et mobilité : le sens de la proximité}

Pour un premier groupe, les discours des jeunes font état des caractéristiques de l'espace rural de faible densité. Ils décrivent le caractère naturel et agricole de l'espace et ce qui fait défaut : l'absence d'activité de loisirs et d'animation sociale, la faible densité de personnes, le manque de services, l'uniformité de marché professionnel, tout comme la difficulté d'accès à l'emploi, à l'information à cause de l'éloignement. Plusieurs thèmes présents dans les entretiens donnent à voir un espace fermé. Par exemple, l'interconnaissance est mal vécue car elle interfère avec la volonté des jeunes d'évoluer discrètement, elle est vécue comme une surveillance mais elle est reproduite dans la mesure où des relations sociales sont centrées sur le village et ses habitants. L'espace de faible densité est considéré comme un cadre peu pénétré d’influences extérieures, replié sur lui-même. Les discours soulignent le sentiment d'y être coincé et dévalorisé. Les jeunes sont eux marqués par cet espace qui manquerait d'horizon et qui est vécu comme enfermant.

Leurs pratiques traduisent un vécu moins négatif des espaces de faible densité parce qu'ils ont une vie quotidienne animée et bien remplie. Ils se livrent en effet à de nombreuses activités entre eux, leur socialité primaire est intense et nous avons vu peu de jeunes livrés à l'ennui et désœuvrés. Différents cercles tels que la famille, les amis, les petits amis, renforcent l'attachement local. Ces pratiques laissent penser que les jeunes sont donc présents, actifs et visibles dans les territoires ruraux. La nuance est de mise, car si présence il y a, elle est décalée. En effet, les jeunes se retrouvent dans des lieux exclusifs (le banc, l'arrêt de bus) qu'ils s'approprient fortement et dont ils excluent les adultes et ceux qui n'appartiennent pas à leur cercle de socialité primaire. Ils se donnent rendez-vous dans des lieux cachés et difficiles d'accès (lacs, bois, ruines) dans lesquels on (l'enquêteur) n'accède pas sans être introduit. Lors d'événement important de la vie du village (fêtes locales, festivals, expositions), les jeunes occupent aussi des lieux qui sont à l'écart des espaces publics (sur le parking du pub ou derrière la salle des fêtes pendant des événements). Ils sont finalement peu visibles dans l'espace public et ne participent que très peu à la vie locale.

La mobilité n'est faite que de déplacements nécessaires et contraignants. Elle s'organise alors autour des lieux facilement accessibles dans un périmètre continu, plus ou moins étendu, centré sur le lieu de résidence. Peu marquée par la multi-appartenance territoriale, leur mobilité l'est en revanche par de nombreux déplacements entre des localités voisines. Ils n'ont pas intégré, dans leurs pratiques de l'espace, l'idée de mobilité géographique à une autre échelle que locale : celle du nord du département pour le Périgord Vert et celle du bassin de vie pour le Comté Galway. La mobilité leur permet de mâ̂triser un territoire local, mais la migration, à cause de l'attachement au local, ou de la perte de repères connus qu'elle engendre, ne fait pas partie de leur mode de vie (Gambino, 2010). C'est ainsi que se délimite un territoire, continu, aréolaire, construit dans une dialectique entre enfermement local et circulation intense dans un périmètre bien identifié. Le territoire dessiné par leur mobilité géographique se rapproche d'un territoire circulatoire.

Généralement, ce sont les adolescents et jeunes adultes ayant peu l'occasion de découvrir d'autres espaces qui pratiquent majoritairement cette mobilité locale. Moins scolarisés et dans une situation économique souvent précaire, leur insertion sociale et professionnelle doit beaucoup à leurs réseaux familiaux ou à leurs connaissances.

\section{Ruralité et instabilité résidentielle}

Dans les discours d'un deuxième groupe apparaissent d'autres caractéristiques de l'espace de faible densité, les jeunes décrivent l'attrait que leur département exerce sur les étrangers. Les jeunes du Comté Galway notamment évoquent la construction récente de lotissements ou d'infrastructures de transports. Ils se livrent aussi à un inventaire de lieux, d'activités, de services et aussi de caractères forts : the community spirit, la tranquillité, la convivialité. Deux thèmes font 
ressortir des composantes, selon eux, de la campagne. D'abord la nature : les jeunes font abondamment référence au décor, au paysage, à la beauté de l'environnement qui les entoure. Ensuite, le thème de la qualité se décline à travers la qualité de l'éducation (petites classes, proximité avec les enseignants) et la qualité des produits et la qualité de la vie. Celle-ci tient aussi à la présence des habitants et à l'entente qui peut exister entre eux. Ils trouvent que l'éloignement caractérise ces espaces. Mais ils le vivent comme une protection contre la ville. Les représentations des jeunes se construisent autour d'une opposition entre les attributs de la ville, lieu où les jeunes peuvent se sentir seuls et ceux de la campagne, lieu où les jeunes se sentent protégés. L'opposition des discours sur la ville et sur la campagne fait ressortir le rôle que les jeunes donnent aux espaces de faible densité : celui d'un refuge.

Les pratiques sont structurées tant en France qu'en République d'Irlande, autour des loisirs qui occupent leur temps libre et qui permettent d'avoir une sociabilité intense, de conserver des liens dans le village et d'en forger de nouveaux en ville. Les jeunes font état d'un capital relationnel nourri, au sein duquel peuvent se distinguer des amis proches, des amis d'enfance, des connaissances, des relations de travail, des copains de sortie... Ils tissent des liens sociaux à la fois forts et superficiels autour d'un diptyque lieu de vie d'origine - lieu de travail ou de scolarité. Leurs pratiques répondent véritablement à une stratégie identitaire intériorisant les valeurs de la "société des 35 heures », de loisirs, temps libre, réussite sociale, individualisme, accession à la propriété (Viard, 2002). Elles témoignent d'une appropriation forte de sphères personnelles et privées (maison des parents, maison des amis). L'attachement est également incontestable pour les différents lieux du village propices à leurs pratiques : le club de sport (en particulier pour les garçons) en Irlande et le café pour les jeunes en France.

La mobilité dans leur cas se caractérise par une instabilité résidentielle, ponctuée par des déplacements fréquents entre leur lieu d'appartenance dans l'espace rural et leur lieu de résidence en ville. Deux lieux de résidence coexistent en complémentarité. Un espace d'origine et d'appartenance identitaire stable, représenté par l'espace rural de faible densité, où se trouve la maison des parents, et qui renvoie à la normalité de la vie, à leur univers familier. Il se double d'un espace secondaire, lieu de réalisation sociale, investi par choix ou par nécessité. Ils vont et viennent entre ces lieux de vie au sein desquels ils recréent des sociabilités distinctes, se forgent des compétences et adoptent des pratiques spécifiques. Ce mouvement pendulaire permet l'appropriation et le marquage d'un territoire qui se construit dans l'itération des allersretours entre la ville et le village (Gambino, 2010). Pour mieux vivre et tirer parti de l'incertitude de la jeunesse, ils mettent en place une décohabitation et jouent sur deux mondes différents. Par cette mobilité s'affirme davantage la figure du territoire à la carte, propre au mode de vie contemporain.

Cette mobilité alternante touche en majorité des étudiants et des jeunes ayant entre 18 et 20 ans, principalement issus des couches moyennes. Les filles y sont plus représentées que les garçons. Leur niveau d'étude, comme celui de leurs parents, est varié mais tous considèrent la poursuite d'études comme un élément de réussite sociale.

\section{La ruralité construite autour de la sédentarité}

Enfin dans un troisième groupe, d'autres caractéristiques de l'espace de faible densité sont mises en avant : l'isolement, la distance auxquels les jeunes sont, selon leurs dires, « habitués ». La notion d'habitude souligne combien l'isolement n'est pas interprété, au contraire des types précédents, en termes de contrainte ou de protection. Le manque, notamment de formations professionnelles, s'il est un élément différenciant ces espaces, leur donne toutefois des opportunités. En effet, il les pousse à regarder ailleurs, à chercher des lieux de formation, à découvrir d'autres environnements. Pour eux, vivre dans ce type d'espace oblige à se débrouiller, à s'organiser, à anticiper, à développer leurs compétences. Les jeunes se représentent donc l'espace rural de faible densité comme un lieu de réalisation personnelle ou professionnelle car ses caractéristiques posent des défis qui offrent des perspectives aux jeunes. Ils détournent leur description des qualités ou des désavantages liés à la faible densité mais mettent l'accent sur ce que ce cadre de vie a offert : une éducation et une famille. L'espace de faible densité est vécu comme un signe distinctif. Il leur donne des «capabilités » (Sen, 1981) qui les singularisent car leurs lieux de vie, à leurs yeux, ne sont pas des 
lieux quelconques. Les lieux sont vécus comme un « espace des possibles» car ils sont le cadre des projets de vie des jeunes.

Quelles sont les pratiques des jeunes ayant cette représentation? Ils s'occupent à mettre en place un projet de vie ancré territorialement, conserver ou revaloriser un patrimoine, cultiver un jardin, vivre au calme, être présent où ils ont grandi. Le travail constitue un moyen de financer l'installation dans l'espace rural. Il permet de financer les études, de se déplacer, mais ne représente pas la pierre angulaire de leur projet de vie (contrairement aux précédents). La famille, plus précisément le couple, permet de connaître du monde et d'être entouré, constitue un repère et un soutien. Leur sociabilité est cependant mise en œuvre de façon très individuelle et maitrisée. Quant aux loisirs, ils sont aussi moins tournés vers les activités sportives et davantage vers des activités culturelles et associatives qui font l'objet d'un investissement plus fort que celui des jeunes des deux autres types.

À travers leurs pratiques et leurs représentations, on peut voir que les espaces ruraux de faible densité expriment une correspondance entre leur identité et la ruralité. Ici, la mobilité est une condition de réalisation sociale (Gambino, 2010). Elle représente le moyen de vivre selon une logique différente de la majorité des autres jeunes, et constitue une alternative à la vie en ville. Elle est fonctionnelle, entièrement maîtrisée et mise au service d'un projet de vie, à savoir vivre chez soi à la campagne. Elle est rendue possible par le permis de conduire (qui facilite l'autonomie dans le déplacement) et par la situation professionnelle. La mobilité quotidienne est mise au service de la sédentarité, centrée sur la maison et l'espace local élargi auquel les jeunes ruraux s'identifient. Elle est également caractérisée par la mise en relation de nombreux lieux éloignés les uns des autres, chacun correspondant à des rôles différents dans la vie quotidienne : le lieu de résidence, le lieu de travail, le lieu des loisirs et de la sociabilité, le lieu de la famille (la maison des parents et celle d'autres membres de la famille). Le lieu de vie dans son ensemble est donc d'une part ancré sur la maison individuelle ainsi que le village et, d'autre part, éclaté en plusieurs lieux éloignés auxquels sont assignés des fonctions précises (récréative, professionnelle, sociale, etc.).
Les jeunes interrogés qui adoptent cette forme de mobilité sont souvent les plus âgés et les mieux diplômés. Cependant, tous n'ont pas fait des études supérieures. Ils ont en revanche en commun un fort capital culturel et exercent souvent un métier artisanal ou artistique. Leur réseau relationnel privilégie la qualité des liens sociaux à leur nombre. Ils sont issus de familles pour lesquelles vivre en milieu rural fait partie d'un projet de vie, ou de retour, pour ceux qui en étaient originaires.

L'évocation des représentations et des pratiques montre que la mobilité des jeunes ruraux ne peut être seulement interprétée comme la marque d'une désaffection pour la ruralité, ceux-ci restent (quitte à se sentir piégés), reviennent, et/ou s'installent. Ils sont nombreux à concevoir la ruralité comme un lieu correspondant à leurs modes de vie, marqués par la tension entre l'impératif de mobilité et l'aspiration à la sédentarité rurale.

\section{UNE APPROCHE PSYCHOPATHOLO- GIQUE DE L'HABITER RELATIVE AUX PRATIQUES ET AUX AFFECTS EXPRIMÉS PAR LES JEUNES}

Parallèlement à cette approche géographique de l'habiter des jeunes, nous avons souhaité compléter ce premier éclairage par le recours aux sciences humaines dites « cliniques ». En effet, derrière ce qui est manifeste et repérable grâce aux méthodes du géographe, il existe un repérage qui doit se faire entre les lignes des actes posés par les jeunes. Ce repérage est rendu possible grâce à l'organisation méthodique d'une interaction avec le jeune, un «cheminement commun» (Stern, 2003) qui vise à reconstruire les soubassements de la mobilité des jeunes dans leur propre territoire. Comprendre cette mécanique intrapsychique consiste donc à interroger justement une pratique sociale à travers ce qui se joue dans le monde intérieur du jeune et la façon dont cela interagit avec le territoire.

\section{Les sables mouvants comme mobilité décalée}

Il y a plusieurs années de cela, nous avons rencontré Zoé qui souhaitait revenir dans son village natal pour créer un festival culturel pour « jeune public ». Elle faisait ses études dans une autre région mais, 
elle voulait réinvestir ses compétences dans son village. Elle s'est mise au service d'une association locale pour inventer un festival qui existe désormais depuis plusieurs années et qui permet, tous les ans, à des enfants qui vivent en milieu rural, d'accéder à des rencontres de qualité avec des artistes locaux et internationaux. Outre les difficultés et les blocages qu’a pu rencontrer Zoé, nous avons, de notre côté, suivi cette initiative à travers une aide et une possibilité d'expression libre au sein d'une brochure départementale. Cette initiative s'est donc inscrite dans la durée, elle a même élargi son rayonnement territorial mais parallèlement, s'est développé une géographie pulsionnelle que nous n'aurions pas pu saisir sans une approche longitudinale du parcours de Zoé. En effet, derrière ce qui est manifeste et sophistiqué (la création d'un festival pour « jeune public » en milieu rural ancré dans une unité de temps et de lieux), nous avons progressivement découvert un envers du décor qui correspond à la façon dont Zoé gère ses entrées et ses sorties du temps social (temps de travail, de l'éducation, familial, temps libre), ce que Boccara appelle le «trou noir du social» (1994). Ce que nous avons pu constater avec Zoé, c'est qu'il existe bien une suspension par le sujet du temps social pour travailler la relation avec son univers pulsionnel et affectif, sorte d'aller-retour au sein de ce que nous appelons « l'arrière-boutique ». Contrairement à ce que l'on pourrait penser, cette arrière-boutique, n'est pas seulement un refuge mais une scène ouverte où se jouent des conflits intrapsychiques. Ainsi, à partir de la matière brute que nous a laissé Zoé (un festival, des partenaires, des prises de paroles, un article etc.), nous l'avons, de nouveau, sollicitée notamment à travers ses mots et la justification de sa pratique.

Bien évidemment, ce questionnement a eu lieu plusieurs années plus tard grâce au travail clinique que nous engageons avec chaque jeune et il s'est construit dans une progression de l'analyse : des échanges de courriels, des rencontres ponctuelles puis des entretiens. Dans l'entre-temps, Zoé s'est déplacée entre la Bretagne, la région Rhône-Alpes, la Bosnie, la Turquie et surtout, fait intéressant, elle a fini par revenir dans le lieu du festival. C'est donc à partir ce qu'elle avait écrit plusieurs années auparavant qu'a démarré notre travail avec Zoé. Il est complexe de rendre compte ici ce que représente cette " géographie pulsionnelle », mais, l'envers du décor, le soubassement du festival pourrait-on dire, nous a conduit à analyser le rapport que Zoé entretient entre son attachement au territoire, ses déplacements et ses retours ponctuels. Ainsi, lorsque Zoé initie son festival, nous sommes à mille lieux de savoir qu'elle venait d'avorter. Cette expérience de la douleur sera donc un premier moyen pour Zoé d'affronter ses conflits, sa tristesse avec la réalité de la mise en place d'un festival (son « bébé » ditelle). Bien entendu, Zoé nous dira que l'absence du père sera, selon elle, aussi un fil conducteur de sa recherche mais, c'est à l'occasion d'un long entretien avec elle que nous apprendrons qu'il s'est passé quelque chose dans son village natal (le lieu du festival) : Zoé a été victime d'attouchements sexuels durant son enfance. Pour ne pas sombrer (plusieurs tentatives de suicide à l'adolescence), Zoé passe son temps à s'engager dans ce qu'elle appelle des «situations rocambolesques » et des temps de recherche (des thérapies, l'écriture d'un journal, des dialogues avec des personnes ressources). Ces «sables mouvants », comme elle nous l'indique, lui sont indispensables pour avoir une certaine maîtrise de l'équilibre. Zoé n'est donc jamais là où la logique sociale voudrait qu'elle soit, mais cela ne l'empêche pas de s'impliquer dans la vie publique locale. Ici, la suspension du temps social n'est pas synonyme de renoncement ou bien de déni du social mais de mise à distance vitale pour accéder à la connaissance.

Cet exemple emprunté à la clinique montre que derrière ces déplacements géographiques entre le village d'enfance de Zoé et des lieux éloignés, il existe un autre type de déplacements dont le moteur est éminemment pulsionnel. Malgré un trouble psychologique, une situation familiale avec l'absence du père, cette jeune femme arrive à organiser et animer un festival et à assumer ce qui pour la famille et les pouvoirs publics pourrait ressembler à un parcours à risques. Ainsi, même si, comme disent ses proches avec un profond respect, il est parfois « difficile» de suivre Zoé, elle laisse un festival, une trace de son passage en tant que « lieu unitaire où peut s'effectuer le rassemblement du divers » (Starobinski, 1993).

\section{L'errance comme autre forme de mobilité}

De la même manière, une expérimentation de l'errance n'est pas forcément dépendante des conditions sociales et économiques. Cette forme de 
mobilité radicale est une autre manière d'aborder les modes « d'accessibilité socio-cognitive de l'espace géographique » (Ramadier, 2011). C'est donc volontairement, que nous avons souhaité faire référence à l'expérience de Martha qui, lorsque nous l'avons rencontrée, était « socialement étiquetée » comme jeune «Sans Domicile Fixe» alors que sa trajectoire et sa position nous conduisent à lire entre les lignes de nos classifications. Martha est issue d'une famille d'ouvriers avec un père qui était à l'origine routier puis artisan terrassier et une mère ouvrière dans le textile (mécanicienne en confection) qui finira sa carrière dans la fraisiculture. À la suite de la mort de son père, alors qu'elle avait treize ans, Martha va basculer dans une fuite en avant, hors de sa famille, pour entrer progressivement en errance avec « ses amis de la rue ». Au fond, depuis toujours, elle ne s'accommode pas de cette vie de famille au sein de laquelle les parents travaillent énormément sans prendre vraiment le temps de se parler. Ainsi, malgré la mort, la violence du couple parental, une vie peu expressive au sein de la famille, l'omniprésence de la religion, la violence avec son partenaire... cela n'empêche pas Martha de reconstituer un idéal à l'extérieur, dans son implication en tant que mère, au travail, dans les associations, avec ses amis. C'est donc à partir du lycée, précisément en première, qu'elle décide d'entamer une rupture avec sa formation initiale en décidant d'aller vivre avec ses amis dans la rue. Elle se sent, dit-elle, en «insécurité dans le lycée et son internat », comme persécutée par le « regard des autres ». Les questionnements radicaux de Martha ne trouvent pas forcément un écho dans la communauté éducative du lycée et l'orientation qui lui sera proposée (comptabilité) finira par la rendre totalement étrangère à la vie scolaire tandis qu'elle souhaitait poursuivre des études dans le domaine de la littérature. Elle le dit encore aujourd'hui, elle a lancé un défi en mettant fin à son parcours scolaire (en partant elle a dit à ses enseignants : «À bientôt dans la rue! »). Martha s'engage dans une vie de rue tout en conservant un point de chute (un appartement) et un contact avec sa mère. Pour assurer le quotidien, elle fait des petits boulots et elle apprend à ne « pas rester inactive » : dans la rue, selon Martha, il y a ceux qui ne font vraiment rien et il y a ceux qui vont vers les gens pour proposer un service, « rendre un sourire », faire une animation. De cette époque, elle a conservé des amis qu'elle voit encore et dont certains sont toujours dans la rue. Elle décrit ses amis comme étant à la fois des voyageurs et des personnes qui étudient. Dans la rue, elle a rapidement sympathisé avec les plus anciens, les «plus vieux de la zone » car ils sont gentils, partagent et surtout parlent de leurs vies, de leurs voyages. Elle va s'intégrer progressivement à la communauté, connaître les lieux où l'on squatte (les trains condamnés dans les zones de triages), les lieux de rendez-vous réguliers, les personnages sédentaires qui assurent des relations avec les nomades (la «mamie caddy » qui fait ses courses, sollicite une aide pour du portage et qui, en échange, fait des achats pour le groupe). Comme cela est rendu difficile socialement parlant, Martha se sert du no man's land comme d'un bouclier protecteur (comme une défense aurait dit Freud) tout en continuant à prendre du temps (perdre du temps diront ses amis ou ses employeurs) pour continuer ses recherches et ses analyses. Elle va, progressivement, revenir à une vie qui s'équilibre entre l'éducation de son fils, un engagement déterminant au sein d'un projet européen et surtout une vie qui s'assume dans une globalité : Martha, malgré un contexte social et économique difficile, est dans une recherche permanente qui ne dissocie pas les éléments dépressifs d'une construction progressive de sa connaissance.

\section{Les trajectoires et les espaces de l'intérieur}

À travers l'évocation de deux cas cliniques, nous avons voulu montrer l'intérêt que revêt cette forme d'analyse microscopique du social qui entend investiguer une mobilité manifeste à travers ses fondements pulsionnels. En effet, comme on a pu le voir avec les expérimentations de Zoé et de Martha mais aussi par le biais de l'application méthodique d'une clinique de l'éducation, une mobilité latente dresse un autre rapport des jeunes à leur territoire faisant état de leur temporalité qui alterne entre engagement dans la vie publique locale (création d'un festival, projet européen), vie professionnelle et privée (formation, recherche d'emploi, logement, loisirs...) et préoccupation intérieure pour reconstruire une histoire et, finalement, une géographie qui se laisse pénétrer par des éléments affectifs.

Entre le déplacement de Zoé pour, officiellement, revenir dans son village afin de faire son stage pra- 
tique d'animatrice et celui officieux pour tenter de retrouver son agresseur et lui exprimer son malaise, il y a un décalage, un contretemps qu'on ne peut pas voir si nous n'introduisons pas la mise en chantier du jeune pour construire et reconstruire sa propre trajectoire de vie. De la même manière, pour explorer une notion comme la mobilité, il faut dès lors ne pas exercer un « déni de sens » (Bourdieu, 1979) en reléguant d'autres catégories comme celle de l'errance dont on a vu, avec Martha, qu'elles correspondent à des pratiques sociales signifiantes.

D'ailleurs, dans une approche résolument macrosociale, cette hypothèse générale concernant l'état de la jeunesse en France a bien été caractérisée par la sociologue C. Van de Velde (2008) qui introduit, selon nous, une variable tout à fait novatrice pour décrire ce qu'elle appelle le «devenir adulte». Réinitialisant ainsi le questionnement par les sciences humaines des effets de la normativité des politiques publiques, elle redonne, grâce à l'emprunt de la notion du « devenir ", tout son sens à une trajectoire sociale comme celle de Martha ou de Zoé.

\section{Diversité DES MOBILITÉS ET PLACE DES JEUNES}

Dans ce contexte de travail pluridisciplinaire entre la géographie et la psychopathologie, nous avons essayé de montrer que si l'on souhaite aborder une problématique comme la mobilité avec les jeunes, il faut au préalable prendre en compte leurs pratiques et ce qui va avec. Nous constatons que quelle que soit notre posture de recherche, trois tendances se dégagent de la mise en regard de nos travaux respectifs.

La première tendance est que les jeunes habitent leur espace rural selon une logique de circulation. En effet, contrairement à ce qui est souvent exprimé par la communauté des adultes, les jeunes circulent dans leur territoire plus qu'on ne le pense. Tout comme le montre M. Gambino, ils développent une vie relationnelle parfois intense qui est peu visible ou peu repérée parce que cachée ou éloignée du lieu de vie. Si nous savons que les jeunes se mettent systématiquement à l'écart dans la vie publique (en préférant s'installer dans ce qu'ils appellent les « recoins », c'est-à-dire, l'arrière du bistrot ou 20 mètres face à la salle des fêtes où sont les adultes), ils sont souvent ignorés dans le rapport aux territoires du quotidien. Les travaux d'O. Desmesure confortent cette tendance pour ces jeunes qui vont et viennent parfois dans le silence et l'indifférence liés à un drame personnel et pour lesquels il n'existe pas forcément d'espace pour exprimer cette solitude.

La deuxième tendance est que les jeunes sont dans une logique d'ancrage parce que leur cadre de vie peut à la fois les préparer à une future sédentarité tout en représentant une protection face à l'avenir : les territoires font repère y compris pour celles et ceux qui s'engagent loin de chez eux. En revanche, si pour certains jeunes, la nature et le cadre de vie sont une richesse, pour d'autres, ils ne sont qu'épisodes dans un enchaînement psychopathologique au sein duquel une personne ou un groupe de personnes peuvent être la figure de référence.

Enfin, la troisième tendance commune est celle qui contredit le plus le modèle normatif de la mobilité et qui a trait à l'errance. En effet, l'errance est souvent interprétée comme une perte de contrôle de soi ou comme une maladie alors qu'elle représente, selon nous, un versant commun à l'ensemble des jeunes en tant que droit à l'expérimentation.

Ce qui se passe dans les endroits reculés ou dans les espaces favorables à l'expression des jeunes met en lumière une vie intense et une aspiration à la prise d'initiative en tant que mise en chantier même si, bien souvent, ces tâtonnements expriment l'ampleur d'un malaise («nos villages sont morts »). Or, comme nous l'enseigne Montaigne (1962), dans toute forme d'errance, lorsqu'elle est imprimée et exprimée, il y a accès à la connaissance, ce que démontrent à eux seuls les exemples empruntés à la clinique.

Ce travail sur les jeunes met bien en évidence une jeunesse dont la mobilité n'est ni uniforme, ni linéaire et peu compréhensible par une seule approche disciplinaire. Ce résultat est d'autant plus fondamental qu'il montre que vouloir mettre les jeunes «sur des rails » (Van de Velde, 2008) (par la «tyrannie du diplôme », du placement par les Missions Locales, de l'injonction à la mobilité), tout en ignorant les tâtonnements, les balbutiements est contre-productif pour donner du sens à leurs modes d'habiter et concevoir un accompagnement des jeunes. C'est pourquoi nous parlons de contretemps de la jeunesse pour qualifier leurs modes d'habiter.

De façon plus générale, les jeunes constatent une domination du monde adulte dont ils sont dépen- 
dants. Le sentiment de n'avoir aucun contrôle et aucune emprise sur ce monde accentue leur isolement social. Ce ressenti existe par ailleurs pour les jeunes vivants dans les périphéries des villes où se sont déroulées les émeutes en 2005 , où il se conjugue avec un sentiment d'abandon (Avenel, 2006). Étant peu consultés, nous remarquons que les jeunes se dévalorisent. Ils pensent que ce qu'ils font est toujours moins bien que ce que pourraient faire les autres. La dévalorisation les pousse à sousestimer leurs capacités à réaliser leurs projets. Cela crée les conditions pour que se développe chez les jeunes, mais aussi pour l'ensemble des habitants un sentiment de mise à l'écart.

Parallèlement, il ne suffit pas de mettre en place des équipements et des dispositifs pour aménager le territoire, encore faut-il, pour cela penser et pratiquer l'aménagement éducatif de ce territoire. Au moment où justement il est fortement question de remettre la jeunesse au cœur des politiques publiques, nous posons là les limites d'une « pensée d'État » telle que décrite par P. Bourdieu qui mesure le développement des territoires au prorata de la quantité de dispositifs mis en œuvre tout en continuant à morceler les approches entre les administrations. Dès lors, nous voyons bien que lorsque les partenaires territoriaux se mettent en « ordre de bataille » pour aménager des espaces de transition et de transmission, il s'agit de faire appel non plus uniquement à la capacité de production d'une société mais à sa capacité d'intégration.

Enfin, si nous sommes tout à fait d'accord pour dire qu'il existe désormais des clivages importants entre les jeunes et la société des adultes, nous ne sommes pas certains que les voies uniques des sciences humaines (qu'elles soient cliniques ou non) seront efficaces tant qu'elles seront séparées, elles aussi, des objets qu'elles prétendent investiguer. Par conséquent, accepter de faire des recherches au sujet des jeunes, c'est aussi prendre le risque de pouvoir entrer dans une relation éducative, signifiant ainsi au chercheur qu'il n'est pas qu'un simple observateur. De la même manière, cela implique un rapport de collaboration étroite avec les partenaires éducatifs qui va bien au-delà de l'intervention professorale, c'est-à-dire, un engagement dans un collectif de travail qui s'équilibre entre travail théorique et participation, dans le temps et dans le territoire.

\section{CONCLUSION : LES JEUNES RURAUX, UNE APPROPRIATION DÉCALÉE DE LA MOBILITÉ}

On ne peut pas faire l'impasse aujourd'hui d'une réflexion qui vise à déconstruire cette notion de mobilité devenue, au fil du temps, une sorte d'injonction paradoxale entre un encouragement social à la mobilité (Le Breton, 2005) et une vie qui se sédentarise de plus en plus (Chauvel, 1998). On pourrait, à cet égard, mettre en parallèle deux manifestations de la mobilité avec, d'un côté, celle des camping-cars souvent utilisés par les retraités, illustrant parfaitement cette forme de "mobilité programmée » et, d'un autre côté, celle des mobilités plurielles exposées ici mais aussi décrites par l'ethnologue G. Loiseau (2012) qui, elle, évoque les «tares de la mobilité » pour ces jeunes qui vivent désormais dans les yourtes, tipis, caravanes, cabanes, camions ou autres habitats légers et mobiles.

Comme nous venons de le voir, face à ce que d'aucuns appellent la « souffrance générationnelle » (Chauvel, 1998), il nous a semblé nécessaire de repositionner la notion de mobilité eu égard aux contretemps de la jeunesse mais aussi, à travers la prise en compte de ce qui se révèle être des trajectoires atypiques. C'est aussi ce que confirment P. Loncle et V. Muniglia (2010) en indiquant justement que « les trajectoires des jeunes vers l'âge adulte se présentent avant tout comme étendues, peu linéaires et empreintes de complexité et de réversibilité ».

Par ailleurs, les solutions pour faciliter la mobilité des jeunes ruraux ne sont pas uniquement liées aux transports et aux conditions matérielles de vie, mais aussi et surtout aux conditions sociales et économiques d'usage de leur «motilité » (Kaufmann, Jemelin, 2004 ; Kaufman, Widemer, 2005), c'està-dire de leur capacité à s'approprier la mobilité comme espace d'expérimentation et de connaissance.

Contre « une vision événementielle des problèmes sociaux » (Ebersold, 2013) nous avons voulu montrer, à travers une analyse fouillée des pratiques des jeunes, que la mobilité n'est pas que l'affaire des jeunes mais aussi celle des pouvoirs publics pour introduire le questionnement de la «motilité territoriale », formule inédite qui pourrait désigner, la capacité des partenaires à élaborer une stratégie commune 
notamment à partir d'un repérage établi par un collectif de travail pluridisciplinaire autour des jeunes et de leurs pratiques. C'est en rendant plus favorables les conditions faites aux jeunes dans tous les territoires que les politiques publiques pourront les aider à réussir leur transition vers l'âge adulte.

\section{Bibliographie}

Avenel C., 2006. Les émeutiers de la politique de la ville. Des espoirs d'intégration aux désespoirs d'insertion, Mouvements, Vol. 44-2, p. 36-44.

Besse J.-M., 2013. Habiter, un monde à mon image, Paris, Flammarion, $250 \mathrm{p}$.

Bion W.-R., 1979. Aux sources de l'expérience, Paris, PUF, 137 p.

Boccara M., 2002. La part animale de l'homme, Paris, Anthropos, $148 \mathrm{p}$.

Boccara M., 1994. Le trou noir du social ou les fondements mythiques de la société humaine, in Assoun P-L., ZAFiroPOulos M., La règle sociale et son au-delà inconscient, Paris, Anthropos, p. 167-190.

Bourdieu P., 1979. La distinction, Paris, Minuit, p. 545.

Bronner L., 2007. Les jeunes ruraux ont le sentiment que leurs « galères » n’intéressent personne, Le Monde, le $1^{\text {er }}$ février.

Cifali M., 1996. Transmission de l'expérience, entre parole et écriture, Education permanente, Paris, n $127,1996-2$, p. 183-200.

Chauvel L., 1998. Le destin des générations. Structure sociale et cohortes en France du XXe siècle, Paris, PUF, 301 p.

De Certeau M., 1990. Linvention du quotidien, I-Arts de faire, Introduction générale, Paris, rééd. Gallimard/Folio Essais, $\mathrm{n}^{\circ} 146$, réédition 2002, $316 \mathrm{p}$.

De Certeau M., 1994. La Prise de parole. Et autres écrits politiques, Paris, éd. établie et présentée par Luce Giard, Seuil, $216 \mathrm{p}$.

Desmesure O., 2011 a. Cultures des jeunes : aux sources pulsionnelles de la connaissance, Thèse de Doctorat en Psychopathologie et psychanalyse, Université Denis-Diderot Paris VII, Sorbonne-Cité, 492 p.

Desmesure O., 201 lb. Les initiatives des jeunes comme autre forme d'accès à la connaissance, $P O U R, \mathrm{n}^{\circ} 211$, p. 75-82.

Detienne M., 1981. L'invention de la mythologie, Paris, Gallimard, $256 \mathrm{p}$.

Ebersold S., 2013. "Préface ", in Cordazzo P., Fichet B. (dir.), Transition, passage en sciences sociales, p. 7-9.

Gambino M., 2008. Vivre dans les espaces de faible densité de population, pratiques et représentations des jeunes dans le Périgord Vert (France) et le Rural Galway (République d'Irlande), Thèse de Doctorat de Géographe, université de Toulouse II, $374 \mathrm{p}$.

Gambino M., 2010. Les mobilités géographiques des jeunes dans les espaces ruraux de faible densité, Note d'analyse
Prospective et Évaluation, $\mathrm{n}^{\circ} 22$, Centre d'études et de prospective, [http://www.agreste.agriculture.gouv.fr/IMG/ pdf_analyse221006.pdf].

Gambino M., 2011. Pratiques de jeunes et participation à la vie locale : regards croisés France-Irlande, POUR, n 211 , p. 177-185.

Galland O., Lambert Y., 1993. Les jeunes ruraux, Paris, INRA, L'Harmattan, Alternatives Rurales, 253 p.

Guetat-Bernard H., 2007. Développement, mobilités spatiales, rapports de genre: une lecture des dynamiques des ruralités contemporaines (Inde du sud, Ouest Cameroun, Amazonie brésilienne), mémoire d'HRD, université Toulouse 2 le Mirail, UFR Sciences Espaces Sociétés, Département de géographie, laboratoire Dynamiques Rurales, 3 tomes. Tome 3, 384 p.

Kaufmann V., Jemelin C., 2004. La motilité, une forme de capital permettant d'éviter les irréversibilités socio-spatiales, Communication au colloque Espaces et sociétés aujourd'hui. La géographie sociale dans les sciences et dans l'action, Rennes, 21-22 octobre 2004, 10 p. (document disponible en ligne, [www.univ-lemans.fr/lettres/eso/evenements/ contributions_10_2004:kv.pdf]).

Kaufmann V., Widmer E., 2005. L'acquisition de la motilité au sein des familles - État de la question et hypothèses de recherche, Espaces et Sociétés, Vol. 120-121, p. 199-217.

Le Breton E., 2005. Bouger pour s'en sortir. Mobilité quotidienne et intégration sociale, Paris, Armand Colin, 256 p.

Lazzarotti O., 2006. Habiter. La condition géographique, Paris, Belin, 287 p.

Loiseau G. (coll.), 2012. Habitat mobile, histoire d'un apprivoisement, in Jаnnot V., Gillet P., Regards croisés sur l'habitat léger/mobile, Relier, p. 20-25.

Loncle P. Muniglia V., 2010. Les catégorisations de la jeunesse en Europe au regard de l'action publique, Politiques sociales et familiales, $\mathrm{n}^{\circ} 102$, p. 9-19.

Montaigne M., 1962. Euvres complètes, Paris, Gallimard, $1256 \mathrm{p}$.

Ramadier T., 2011 . L'accessibilité socio-cognitive, Communication au colloque Mobilité spatiales et ressources métropolitaine : l'accessibilité en questions, Grenoble, 24-25 mars 2011 , AISLF, $12 \mathrm{p}$.

RéNAHY N., 2005. Les gars du coin. Enquête sur une jeunesse rurale, Paris, La Découverte, 294 p.

SEn A., 1981. Poverty and Famines: An Essay on Entitlement and Deprivation, Oxford, Oxford University Press, 272 p.

Starobinski J., 1993. Montaigne en mowvement, Paris, Gallimard, $394 \mathrm{p}$.

Stern D. N., 2003. Le moment présent en psychothérapie, un monde dans un grain de sable, Paris, Odile Jacob, 300 p.

VAn De Velde C., 2008. Devenir adulte. Sociologie comparée de la jeunesse en Europe, Paris, PUF, 278 p.

Viard J., 2002. Le sacre du temps libre: la société des 35 heures, La Tour-d'Aigues, Éditions de L'Aube, 210 p. 